Noname manuscript No.

(will be inserted by the editor)

\title{
Spatio-Temporal Stochastic Modelling (METMAVI)
}

\author{
Raquel Menezes ${ }^{1}$. A. Manuela \\ Gonçalves $^{2}$
}

The VI International Workshop on Spatio-temporal Modelling (METMAVI) took place in Guimarães, Portugal, from 12 to 14 September 2012. The meeting was organised by Raquel Menezes as chair of the Local Organizing Committee. METMA stands for a series of workshops specifically devoted to recent developments in spatio-temporal statistics since 2001. It has been traditionally announced as a satellite conference of The International Environmetrics Society (TIES), promoting the application of spatio-temporal statistical methods in different fields related to environmental sciences. The programme of the 2012 workshop also focused applications to health sciences, in particular with regard to issues concerning epidemiology. At the conference more than 100 research scientists, from 15 different countries, attended the invited and contributed sessions and the fruitful discussions afterwards. The conference schedule, with no parallel sessions, favoured the exchange of ideas and experiences.

Apart from the scientific program of METMAVI, we should not forget the historic atmosphere of Guimarães, making it a perfect venue for a scientific meeting, where reflection and debate could be inspired by the city's legacy. Guimarães, the founding location of the Portuguese Nation, was declared a World Heritage Site in 2001 by UNESCO, and it was nominated the European Capital of Culture in 2012.

This special issue summarizes the main contributions made at METMAVI, related to spatio-temporal methodology illustrated with environmental applications.

\footnotetext{
${ }^{1}$ Department of Mathematics and Applications Research Centre of Mathematics - CMAT

Minho University

E-mail: rmenezes@math.uminho.pt

2 Department of Mathematics and Applications Research Centre of Mathematics - CMAT

Minho University

E-mail: mneves@math.uminho.pt
} 


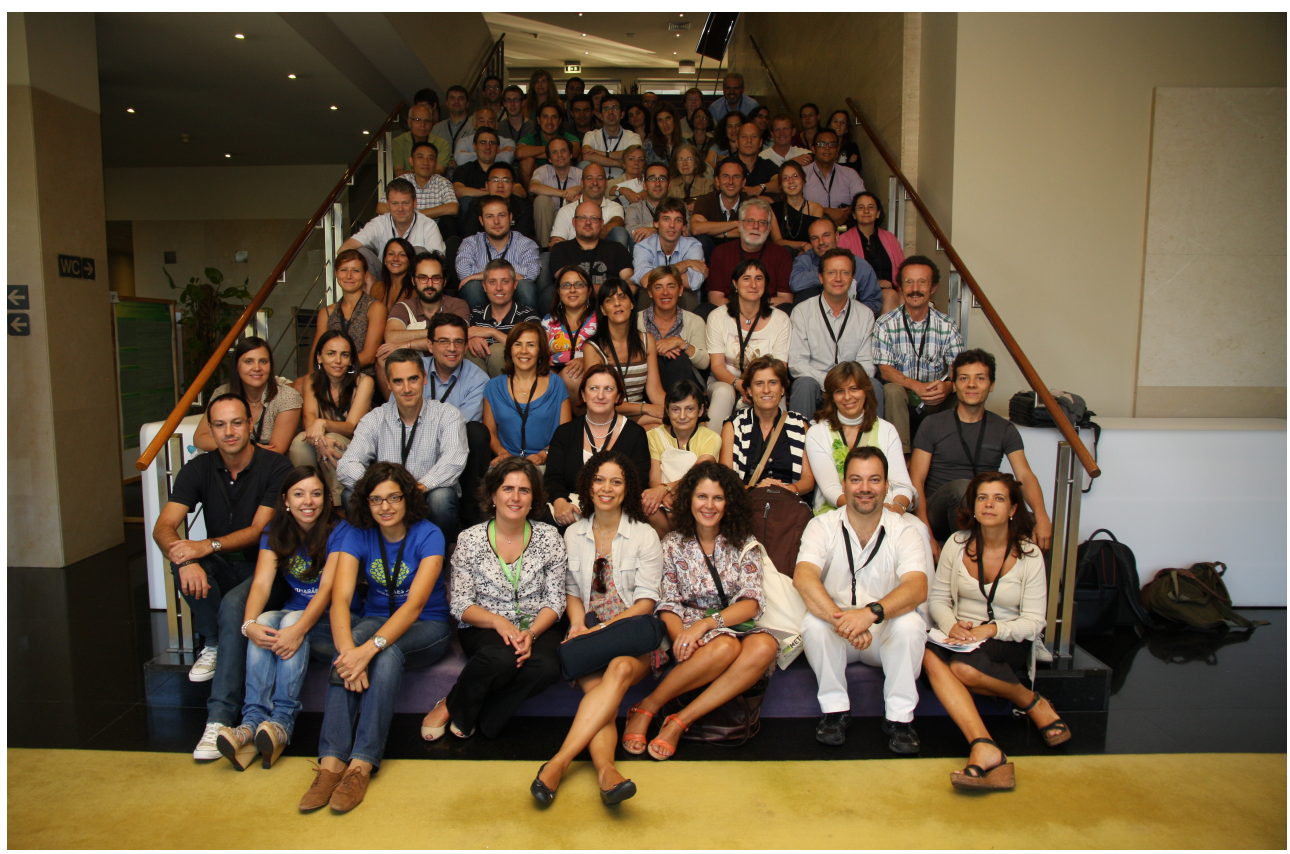

Angulo and Esquivel use methods on entropy and information to study the spatial and temporal patterns in seismic events data in two regions in Spain, showing the usefulness of adopting these tools to understand the spacetime variability. On the other hand, Ruiz-Medina et al. use a functional data analysis approach to model and do inference with spatio-temporal data. More specifically, a non-parametric estimator of the spatial relative risk surface is derived for the context of spatio-temporal epidemiological data, being the method illustrated in the breast cancer mortality data in Spain. Still in a spatio-temporal framework, Margalho et al. do the assessment of interpolation errors, in the sense of demonstrating the importance of using information from covariates, when available, to achieve a better predictive accuracy. Their motivating example is the monitoring of heavy metals in Portugal using moss species as bioindicators, data come from a survey that is being performed across Europe.

García-Portugués et al. suggest a kernel density estimate based test for independence between a linear variable and a directional (circular or spherical) variable. The selection of the von Mises and the normal kernels allows to obtain an easy to compute expression for the involved statistic and also to derive a resampling approach for test calibration. The new test is applied in the analysis of the relation between the orientation and size of Portuguese wildfires. Proceeding in a non-parametric context, Fernández-Casal and FranciscoFernández propose an approach for approximation of the dependence structure of a spatial process, under the presence of a non-constant trend. The local lin- 
ear method is first used for estimation of the trend function and then for estimation of the semivariogram of the resulting residuals.

Another important topic from METMA workshops is the analysis of spatial extremes. Alonso et al. compare Generalized Pareto models fitted to extreme observations. The authors introduce a testing procedure based on subsampling techniques for testing the equality of Generalized Pareto Distributions related with the excesses of two stationary time series, which may not be considered independently generated. An application example is given through data concerning daily maximum temperature in seventeen regions of Spain.

The idea of spatial reconstruction by proposing a framework for calibrating rainfall hourly data obtained from reflectivity radar measurements, exploiting rain gauge data considered as reference measures, is presented in Bruno et al. They aim to model the radar-rainfall relationship in order to obtain spatial predictions, by means of MCMC algorithms in a Bayesian hierarchical framework. Ignaccolo et al. extend the kriging with external drift to the case of functional geostatistical data, when curves along time are observed. Through a three-step procedure, it becomes possible to use scalar as well as functional exogenous variables in the functional kriging. This is important for many environmental applications where the (functional) response variable depends strongly on exogenous variables. The case study considers daily PM10 concentrations measured by the monitoring network of Piemonte region in Italy.

The original Bootstrap procedures were designed for independent data and an adaptation of these procedures to the spatial setting may have large applicability for addressing a variety of issues. García-Soidán et al. introduce nonparametric Bootstrap approaches that allow us to generate replicates from the available data, at a set of locations selected, by first approximating the joint distribution in a nonparametric way and then randomly drawing samples from it. An application example is given by using biomonitoring data of arsenic pollution in the Central Region of Portugal. Finally, Ceyhan investigates a methodology based on nearest-neighbour contingency tables (NNCTs) to analyse the spatial inter-dependencies of multi-class point patterns. This analysis is based on an omnibus test and on specific tests for the pairwise interaction between the distinct point classes. The author proposes several parameterizations of the segregation and association alternatives and proposes three processes for the two-class association patterns.

To conclude we would like to express our gratitude to referees that collaborate in the edition of this special issue. We are specially grateful to the Editorial Board of Stoch. Environ. Res. Risk Assess. for their support. We hope that these contributions will further enhance the current interest in statistical methods in a spatio-temporal framework.

Raquel Menezes and A. Manuela Gonçalves

Guest Editors 


\section{References}

1. Angulo J.M. and Esquivel F.J. (2014). Structural Complexity in Space-Time Seismic Event Data, Stoch. Environ. Res. Risk Assess.

2. Ruiz-Medina M.D., Espejo R.M., Ugarte M.D. and Militino A.F. (2014). Functional time series analysis of spatiotemporal epidemiological data, Stoch. Environ. Res. Risk Assess.

3. Margalho L., Menezes R. and Sousa I. (2014). Assessing interpolation error for spacetime monitoring data, Stoch. Environ. Res. Risk Assess.

4. García-Portugués E., Barros A.M.G., Crujeiras R.M., González-Manteiga W. and Pereira J.M.C. (2014). A test for directional-linear independence, with applications to wildfire orientation and size, Stoch. Environ. Res. Risk Assess.

5. Fernández-Casal R. and Francisco-Fernández M. (2014). Nonparametric bias-corrected variogram estimation under non-constant trend, Stoch. Environ. Res. Risk Assess.

6. Alonso A.M., Zea Bermudez P. and Scotto M.G. (2014). Comparing Generalized Pareto models fitted to extreme observations: an application to the largest temperatures in Spain, Stoch. Environ. Res. Risk Assess.

7. Bruno F., Cocchi D., Greco F. and Scardovi E. (2014). Spatial reconstruction of rainfall fields from rain gauge and radar data, Stoch. Environ. Res. Risk Assess.

8. Ignaccolo R., Mateu J. and Giraldo R. (2014). Kriging with External Drift for functional data for air quality monitoring, Stoch. Environ. Res. Risk Assess.

9. García-Soidán P., Menezes R. and Rubiños O. (2014). Bootstrap approaches for spatial data, Stoch. Environ. Res. Risk Assess.

10. Ceyhan E. (2014). Simulation and Characterization of Multi-Class Spatial Patterns from Stochastic Point Processes of Randomness, Clustering and Regularity, Stoch. Environ. Res. Risk Assess. 